\title{
Bridging dynamic regimes of segmental relaxation and center-of-mass diffusion in associative protein hydrogels
}

\author{
Ameya Rao $\odot$, Helen Yao $\odot$, and Bradley D. Olsen $\odot^{*}$ \\ Department of Chemical Engineering, Massachusetts Institute of Technology, Cambridge, Massachusetts 02139, USA
}

(Received 28 February 2020; accepted 30 October 2020; published 14 December 2020)

\begin{abstract}
Molecular transport in dense, crowded media is often affected by binding and collisions with obstacles, leading to complex spatial distributions and anomalous diffusion on multiple length scales. Here, neutron spin-echo (NSE) spectroscopy and forced Rayleigh scattering (FRS) were used in tandem to study the interplay between segmental and center-of-mass chain dynamics in the presence of strong binding interactions in a model protein hydrogel. The results provide evidence for several dynamic scaling regimes of gel relaxation behavior with varying length scale, including a caging regime bridging submolecular relaxation and center-of-mass diffusion due to transient binding. On mesoscopic length scales larger than the size of the cage, chains undergo two distinct regimes of apparent superdiffusive scaling, with different power-law exponents, before the onset of terminal Fickian diffusion. The combined NSE and FRS results are interpreted in the context of prior Brownian dynamics simulations of associating star polymers, revealing insight into structural length scales and binding kinetics governing the transition from segmental relaxation to self-diffusion in the protein hydrogel. Finally, single-sticker tracer diffusion measurements were performed to directly probe sticker association/dissociation dynamics within the gel, the results suggesting that cooperative cluster motion may play a role in network relaxation on larger length scales.
\end{abstract}

DOI: 10.1103/PhysRevResearch.2.043369

\section{INTRODUCTION}

Understanding molecular transport in dense, crowded media is a central problem in soft matter and biological physics $[1,2]$. Such systems often contain structural features spanning several length scales, from segmental correlations within individual macromolecules to large supramolecular assemblies formed by physical binding interactions [3-8]. For example, the cell cytoplasm is a dense fluid containing sugars, proteins, and organelles of various characteristic lengths [1,9]. Similar hierarchical structure is also found in materials such as polymer networks [10], microporous materials [11], and colloidal gels [12]. Solute diffusion, especially of macromolecules, in these systems can be affected by binding and collisions with obstacles across a range of length scales, resulting in a complex, non-Gaussian spatial distribution and time-dependent diffusivity [1,6,13-15].

Transient binding between dissolved macromolecules can often introduce long-range connectivity to form reversible networks with remarkable transport properties [3,16-18]. For example, the nuclear pore complex combines high- and lowaffinity interactions to form a space-spanning matrix that facilitates selective protein diffusion into the nucleus $[16,19]$.

\footnotetext{
*Corresponding author: bdolsen@mit.edu

Published by the American Physical Society under the terms of the Creative Commons Attribution 4.0 International license. Further distribution of this work must maintain attribution to the author(s) and the published article's title, journal citation, and DOI.
}

Associating bonds have also been incorporated into artificial protein hydrogels to achieve molecular separations in purely synthetic environments $[17,20,21]$. In reversible polymer networks, junction exchange enables chain self-diffusion, imparting stimuli-responsiveness and self-healing abilities [22-26]. In all such systems, understanding how binding interactions affect molecular relaxation and diffusion across time scales is essential to their design and remains an open problem [27-30].

Prior work has shown dynamics in associative systems to be largely controlled by the kinetics of the binding groups, or stickers [30-32]. On time scales smaller than the bond lifetime, molecular relaxation occurs through submolecular fluctuations, whereas on larger time scales, multisticker macromolecules undergo various modes of diffusion including walking, hopping, and cooperative cluster motion [20,28,33-35]. While an average of these modes results in long-time Fickian self-diffusion, recent studies have shown that transitions between the different modes can cause apparent superdiffusive scaling on length scales 100-1000 times the molecular size $[36,37]$. However, these experiments have been limited to diffusion length scales greater than 30 times the molecular radius of gyration, limiting their ability to verify theoretical predictions [34] of smaller length-scale regimes. In addition, few experimental studies have explored the relationship between submolecular relaxation and mesoscale diffusion in associative macromolecular systems, processes relevant to a range of phenomena in biophysical $[16,35,38$ $40]$ and soft matter [30,41-43] systems.

In this work, neutron spin-echo spectroscopy and forced Rayleigh scattering are used in tandem to study the interplay 
(a) P coiled-coil mm APQMLRELQETNAALQDVRELL RQQVKEITFLKNTVMESDASG
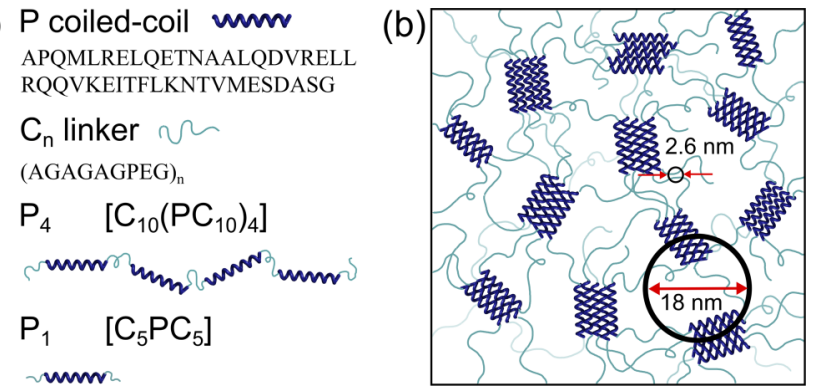

FIG. 1. (a) Amino acid sequences and structures of the coiledcoil $(P)$ and flexible linker $\left(C_{10}\right)$ domains composing proteins $P_{4}$ and $P_{1}$. The molar masses of $P_{4}$ and $P_{1}$ are 62.8 and $16.6 \mathrm{kDa}$, respectively. (b) Structure of the $P_{4}$ network consisting of pentavalent junctions formed by coiled-coil association in parallel orientation. Characteristic length scales $d_{0}=18 \pm 2 \mathrm{~nm}$ and $\xi=2.6 \pm$ $0.2 \mathrm{~nm}$ are obtained from small-angle neutron scattering (SANS) measurements.

between segmental and center-of-mass dynamics on length scales spanning $5 \mathrm{~nm}-50 \mu \mathrm{m}$ in a model associative protein hydrogel. The results reveal several scaling regimes between the characteristic relaxation time and the length scale probed, including a caging regime bridging submolecular relaxation and center-of-mass diffusion and two distinct regimes of apparent superdiffusion on mesoscopic length scales before terminal Fickian diffusion. Comparing the results to recent Brownian dynamics simulations of associating star polymers [34] reveals the role of structural length scales and sticker binding kinetics in governing caging in the associative gel. Finally, single-sticker tracer diffusion measurements are performed to directly probe sticker association dynamics within the network, the results suggesting the importance of cooperative diffusion of multichain clusters in gel relaxation on longer length scales.

\section{EXPERIMENT}

Protein synthesis and purification. $P_{4}$ and $P_{4}$-cys are proteins containing four coiled-coil domains $(P)$ connected by flexible polyelectrolyte linkers $\left(C_{10}\right)$ (see Fig. 1$)$. $P_{4}$-cys differs from $P_{4}$ by the inclusion of a single cysteine (cys) residue near the $C$-terminus that enables site-specific labeling with a photochromic dye via thiol-ene chemistry for forced Rayleigh scattering measurements. The encoding genes, amino acid sequences, and synthetic protocols for $P_{4}$ and $P_{4}$-cys have been previously reported [7]. The genes were cloned into the pQE9 plasmid (Qiagen) with ampicillin resistance and transformed into the Escherichia coli (E. coli) SG13009 cell line, which has the pREP4 repressor plasmid with kanamycin resistance. The transformed cells were grown on LB-agar plates with kanamycin and ampicillin at $37^{\circ} \mathrm{C}$ for $8-16 \mathrm{~h}$. Single colonies were then used to seed starter cultures, which were grown in $50 \mathrm{~mL}$ of LB media with $50 \mu \mathrm{g} / \mathrm{mL}$ kanamycin and $100 \mu \mathrm{g} / \mathrm{mL}$ ampicillin in baffled shake-flasks at $37^{\circ} \mathrm{C}$ for $8-16 \mathrm{~h}$. The starter culture $(50 \mathrm{~mL})$ was used to inoculate $5 \mathrm{~L}$ of TB expression media supplemented with $50 \mu \mathrm{g} / \mathrm{mL}$ kanamycin and $100 \mu \mathrm{g} / \mathrm{mL}$ ampicillin. Cells were grown at $37^{\circ} \mathrm{C}$ and induced with $1 \mathrm{mM}$ isopropyl- $\beta$-D-thiogalactoside
(IPTG) once the expression media reached an $\mathrm{OD}_{600}$ of 0.8 1.0. After induction, cells were allowed to express protein at $37^{\circ} \mathrm{C}$ for an additional $6-8 \mathrm{~h}$. Cells were harvested by centrifugation, resuspended in $250 \mathrm{~mL}$ lysis buffer $(10 \mathrm{mM}$ Tris, $1 \mathrm{mM}$ ethylenediaminetetraacetic acid, $100 \mathrm{mM} \mathrm{NaCl}$,

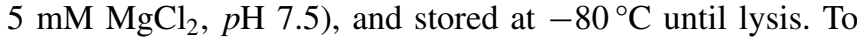
lyse the cells, the cell suspension was thawed, after which $100 \mathrm{mg}$ lysozyme/L expression was dissolved in the cell suspension and rocked for $30-60 \mathrm{~min}$ at $4{ }^{\circ} \mathrm{C}$. The cell suspension was then sonicated on ice in 2-3 cycles of $10 \mathrm{~min}$. The sonicated mixture was then clarified by centrifugation at $26775 \times g$ at $4{ }^{\circ} \mathrm{C}$ for $60 \mathrm{~min}$, after which the supernatant containing the crude protein was denatured by adding $8 \mathrm{M}$ urea and $100 \mathrm{mM}$ monosodium phosphate. The crude protein in the clarified lysate was then purified using two rounds of ammonium sulfate precipitation. In the first round, $20 \%(\mathrm{w} / \mathrm{v})$ ammonium sulfate was added, and the mixture was incubated at $37^{\circ} \mathrm{C}$ with shaking (200-225 rpm) for $2 \mathrm{~h}$. The protein suspension was then centrifuged at $37^{\circ} \mathrm{C}$ for $1 \mathrm{~h}$ at 26775 $\times g$, and the pellet containing the impurities was discarded. For the second round, an additional 15\% (w/v) ammonium sulfate was added to the supernatant, incubated at $37^{\circ} \mathrm{C}$ with shaking for $1.5 \mathrm{~h}$, and centrifuged at $37^{\circ} \mathrm{C}$ for $1 \mathrm{~h}$ at $26775 \times$ $g$. After the second precipitation step, the pellet containing the protein was dissolved overnight at $4{ }^{\circ} \mathrm{C}$ in a buffer containing $20 \mathrm{mM}$ Tris and $6 \mathrm{M}$ urea $(\mathrm{pH} 8)$ and then dialyzed 5 times against ultrapure water (Milli-Q) with at least $3 \mathrm{~h}$ between each water change. $P_{4}$ and $P_{4}$-cys were further purified by anion exchange chromatography on a fast protein liquid chromatography (FPLC) system (GE ÄKTA), using two 5-mL HiTrap Q Sepharose HP columns (GE Healthcare) in series. Proteins were first dissolved in a denaturing buffer containing $6 \mathrm{M}$ urea and $20 \mathrm{mM}$ Tris $(p \mathrm{H} 8)$, loaded onto the column, and eluted by increasing the concentration of $\mathrm{NaCl}$ in the buffer in sequential linear gradients of $0-0.2 \mathrm{M}$ (32 column volumes, $\mathrm{CV}$, with $1 \mathrm{CV}=5 \mathrm{~mL}$ ) followed by $0.2-0.5 \mathrm{M}$ (10 CV). Pure fractions were dialyzed 7-10 times against ultrapure water and lyophilized for 2-3 days until dry. The final yields of pure $P_{4}$ and $P_{4}$-cys were 306 and $36 \mathrm{mg}$ per $\mathrm{L}$ of expression, respectively. Protein purity and molecular weight were confirmed with sodium dodecyl sulfate polyacrylamide gel electrophoresis (SDS-PAGE) (see Fig. S1 of the Supplemental Material [44]) and time-of-flight matrix-assisted laser desorption/ionization (MALDI) mass spectrometry (see Fig. S3 [44]).

For use in neutron spin-echo experiments, a deuterated analog of the protein $P_{4}$ (denoted $d P_{4}$ ) was synthesized by expression in E. coli SG13009 in M9 minimal media containing a deuterated source of amino acids (97\% D Celtone base powder, Cambridge Isotope Laboratories) and $99.8 \% \mathrm{D}$ microbiologically tested deuterium oxide (Cambridge Isotope Laboratories) [45]. Cells were grown at $37^{\circ} \mathrm{C}$ before being induced at $\mathrm{OD}_{600}=0.5-0.6$ after $15 \mathrm{~h}$ of growth in $1 \mathrm{~L}$ of M9 supplemented with ampicillin and kanamycin. Expression was performed at $25^{\circ} \mathrm{C}$ until the death phase was reached around $57 \mathrm{~h}$ after induction. $d P_{4}$ was harvested by centrifugation and purified by ammonium sulfate precipitation and anion exchange chromatography following similar protocols to $P_{4}$ and $P_{4}$-cys. However, for $d P_{4}$, the two cycles of ammonium sulfate precipitation were performed with $13 \%$ and $15 \% \mathrm{w} / \mathrm{v}$ 
ammonium sulfate in each respective step. Anion exchange chromatography was performed in the same denaturing buffer (6 $\mathrm{M}$ urea, $20 \mathrm{mM}$ Tris, $p \mathrm{H} 8$ ) but with a $1-\mathrm{mL}$ HiTrap Q Sepharose HP column (GE Healthcare), and the protein was eluted from the column by increasing the concentration of $\mathrm{NaCl}$ in the buffer in sequential linear gradients of $0-0.2 \mathrm{M}$ $(19 \mathrm{CV}), 0.2-0.4 \mathrm{M}(10 \mathrm{CV})$, and $0.4-1 \mathrm{M}(6 \mathrm{CV})$. The final yield of pure $d P_{4}$ was $4-7 \mathrm{mg}$ per $\mathrm{L}$ expression (purity confirmed by SDS-PAGE; see Fig. S2 [44]) with 78.6\% deuteration and $12.4 \%$ exchangeable protons as measured by MALDI (see Fig. S3 [44]).

For tracer diffusion measurements, a single-sticker analog $P_{1}$-cys was synthesized. $P_{1}$-cys is a protein consisting of one coiled-coil $P$ domain flanked on both sides by $C_{5}$ domains (the same repeating sequence as $C_{10}$ but half the length) (Fig. 1). A single cysteine residue near the $N$-terminus enables dye labeling by thiol-ene chemistry. The full amino acid sequence is provided in the Supplemental Material [44]. The gene for $P_{1}$-cys was obtained from Genscript in the pQE60 vector (Qiagen) and cloned into the $\mathrm{pQE} 9$ vector between the BamHI and HindIII restriction sites using standard molecular cloning protocols. $P_{1}$-cys was expressed by an identical protocol to $P_{4}$ and $P_{4}$-cys and purified by $\mathrm{Ni}^{2+}$-affinity chromatography in denaturing conditions following the protocol reported by Glassman et al. [7]. The final yield of pure $P_{1}$-cys was $11 \mathrm{mg} / \mathrm{L}$ of expression media, and purity was confirmed by SDS-PAGE (see Fig. S4 of the Supplemental Material [44]) and MALDI.

Labeling of $P_{4}$-cys and $P_{1}$-cys with photochromic dyes. Two photochromic dyes, o-nitrostilbene (ONS) and dithienylethene (DTE), were used to site-specifically label $P_{1^{-}}$ cys and $P_{4}$-cys for forced Rayleigh scattering measurements. ONS was used for measurements performed at $488 \mathrm{~nm}$, and DTE was used for measurements performed at $355 \mathrm{~nm}$.

Maleimide-functionalized $o$-nitrostilbene (ONS-M) was synthesized as described previously [46] and conjugated to $P_{4}$-cys using thiol-maleimide coupling as described previously [36] and shown in scheme S1 (Supplemental Material [44]). Briefly, $P_{4}$-cys was dissolved in 3:1 dimethylsulfoxide (DMSO): (10 mM sodium phosphate, $p \mathrm{H} \mathrm{7.5)}$ at a concentration of $1 \mathrm{mg} / \mathrm{mL}$. Next, 20 equiv tris $(2-$ carboxyethyl)phosphine hydrochloride (TCEP) relative to the cysteine residue was added to reduce preformed disulfide bonds, and the mixture was stirred at room temperature for 30 min. Finally, 20 equiv of ONS-M in DMSO was added dropwise, and the mixture was stirred overnight at room temperature in the dark. The mixture was purified by anion exchange chromatography using a HiTrap Q Sepharose HP 5 -mL column (GE Healthcare) to remove free dye, dialyzed against ultrapure water, and lyophilized to yield pure $P_{4}$-ONS. Conjugation of ONS-M to $P_{1}$-cys was performed identically to $P_{4}$-cys except that the reaction buffer also included $6 \mathrm{M}$ urea to increase protein solubility.

Vinyl-functionalized 1,2-bis(2,4-dimethyl-5-phenyl3-thienyl)-3,3,4,4,5,5-hexafluoro-1-cyclopentene (vinyl DTE) was synthesized as described previously [47]. Conjugation to $P_{1}$-cys and $P_{4}$-cys was performed using a radical-initiated thiol-ene reaction as shown in scheme S2 (see the Supplemental Material [44]). In a typical reaction, proteins were dissolved in a 2:1 (vol) mixture of $N, N$-dimethylformamide (DMF): $\quad(6 \quad \mathrm{M}$ urea, 10 $\mathrm{mM}$ sodium phosphate, $p \mathrm{H}$ 7.5) at a concentration of $5 \mathrm{mg} / \mathrm{mL}$. Next, 0.5 equiv of the radical initiator 2,2'-azobis[2-(2-imidazolin-2-yl)propane]dihydrochloride (VA-044) and 10 equiv of vinyl DTE relative to the cysteine residue were added. The reaction mixture was deoxygenated by purging with nitrogen for $30 \mathrm{~min}$ and then heated to $50^{\circ} \mathrm{C}$ for $24 \mathrm{~h}$ under nitrogen. The mixture was purified by dialyzing against ultrapure water and filtering off precipitated free vinyl DTE. To fully remove free dye, the filtrate was then purified by anion exchange chromatography using a HiTrap Q Sepharose HP 5-mL column (GE Healthcare), dialyzed against ultrapure water, and lyophilized. To convert the DTE to its UV-active form for forced Rayleigh scattering (FRS) measurements, $P_{1}$-DTE and $P_{4}$-DTE were irradiated for 1 $\mathrm{h}$ at normal incidence with broadband visible light from an iPhone ${ }^{\circledR} 7$ flashlight.

Rheology. $P_{4}$ gels at 6.5 and $15 \%(\mathrm{w} / \mathrm{v})$ were prepared in $100 \mathrm{mM}$ phosphate buffer in $\mathrm{D}_{2} \mathrm{O}$. Rheology was performed on an Anton Paar MCR 301 rheometer using a cone and plate geometry $\left(25 \mathrm{~mm}, 1^{\circ}\right)$. Silicone oil was used to coat the sides of the geometry to prevent drying during measurement. Gels were heated from 25 to $90^{\circ} \mathrm{C}$ with a ramp rate of $5^{\circ} \mathrm{C} / \mathrm{min}$, held at $90^{\circ} \mathrm{C}$ for $13.5 \mathrm{~min}$, and ramped back down at $5^{\circ} \mathrm{C} / \mathrm{min}$ to $25^{\circ} \mathrm{C}$ to remove thermal history prior to taking frequency sweeps from 100 to $0.001 \mathrm{rad} / \mathrm{s}$. The resulting loss and storage moduli (Fig. S5, Supplemental Material [44]) were compared to those taken for $P_{4}$ gels dissolved in hydrogenated buffer in Ref. [36]. For both concentrations, the crossover frequency is slightly lower for the gels in $\mathrm{D}_{2} \mathrm{O}$ compared to the gels in $\mathrm{H}_{2} \mathrm{O}$, and the plateau moduli are similar for both buffers (Table S2, Supplemental Material [44]).

Small-angle neutron scattering (SANS). Small-angle neutron scattering (SANS) was performed on the EQ-SANS instrument at the Oak Ridge National Lab (ORNL) Spallation Neutron Source (SNS). Two instrument configurations were used to cover a $q$ range of $0.0389-8.10 \mathrm{~nm}^{-1}$. The low- $q$ configuration had a sample-to-detector distance of $4 \mathrm{~m}$, with a wavelength of $12 \AA$; the high- $q$ configuration had a sampleto-detector distance of $2.5 \mathrm{~m}$, with a wavelength of $2.5 \AA$. The EQ-SANS instrument uses a $1 \times 1 \mathrm{~m}^{3} \mathrm{He}$-tube detector with a resolution of $5.5 \times 4.3 \mathrm{~mm}$ to detect scattered neutrons. All data were collected using a $10-\mathrm{mm}$ beam aperture size.

$P_{4}$ hydrogels were prepared at a concentration of $6.5 \%$ (w/v) protein by mixing $P_{4}$ protein with $100 \mathrm{mM}$ sodium phosphate buffer in $\mathrm{D}_{2} \mathrm{O}(99.9 \% \mathrm{D}$, Cambridge Isotopes Laboratories) at $p \mathrm{D}$ 7.6. The blends were allowed to equilibrate overnight at $5{ }^{\circ} \mathrm{C}$ before stirring and centrifuging for $15 \mathrm{~min}$ at $13100 \times g$ and $10^{\circ} \mathrm{C}$. This process was repeated until a homogeneous gel was formed. The hydrogel was spread onto a quartz window and sandwiched between another quartz window with a $1-\mathrm{mm} \mathrm{Ti}$ spacer. The gel sandwich was held in place using a sealed $\mathrm{Ti}$ demountable cell. The hydrogel was then annealed at $80^{\circ} \mathrm{C}$ for $10 \mathrm{~min}$ to remove thermal history, sterilize the material, and allow bubbles to float to the top of the cell outside of the $10-\mathrm{mm}$ beam aperture. After annealing, the gel was stored at $5^{\circ} \mathrm{C}$ until experimentation. The gel was allowed to equilibrate at $25^{\circ} \mathrm{C}$ for $15 \mathrm{~min}$ before running SANS at the same temperature. SANS intensities were corrected for empty cell scattering and blocked beam 
background and calibrated to an absolute scale using a Porasil silica standard. The data from the two configurations were stitched together after 1D data reduction in MantidPlot by matching overlapping $q$ ranges from the two configurations $\left(0.6-0.7 \mathrm{~nm}^{-1}\right)$; data reduction was done using the EQSANSTableReduce function in MantidPlot [48,49]. Solvent background subtraction was also performed after 1D data reduction, with a volume fraction of $0.05(\mathrm{v} / \mathrm{v})$. Data outside $q$ values of $0.05-6 \mathrm{~nm}^{-1}$ were trimmed due to instrumentrelated distortions at lower and higher $q$.

Neutron spin-echo (NSE) spectroscopy. Neutron spin-echo (NSE) spectroscopy was performed at the NSE spectrometer at the Oak Ridge National Lab (ORNL) Spallation Neutron Source (SNS). Samples were prepared by dissolving $P_{4}$ protein in $100 \mathrm{mM}$ phosphate buffer in $\mathrm{D}_{2} \mathrm{O}$ at $p \mathrm{D} 7.6$ in the same way as described above for SANS. For the $6.5 \% \mathrm{w} / \mathrm{v}$ gel, a small amount $(6.3 \% \mathrm{w} / \mathrm{w})$ of deuterated $P_{4}$ was blended with hydrogenated $P_{4}$ because of a low projected flip ratio of coherent to incoherent signal. However, the experimental flip ratio of the $6.5 \%$ gel was found to be sufficiently high due to strong coherent scattering from the $P_{4}$ network, and thus the $15 \%$ gel was prepared with only hydrogenated $P_{4}$. The inclusion of this small amount of deuterated $P_{4}$ in the $6.5 \%$ gel is unlikely to significantly alter its internal chain dynamics or relative contribution of intramolecular vs intermolecular correlations to the NSE signal, allowing comparison of the NSE results for the $6.5 \%$ and $15 \%$ gels. After gels were formed, they were spread onto aluminum front-loading cells with a path length of $2 \mathrm{~mm}$ and sealed with indium wire. The gels were then annealed at $80^{\circ} \mathrm{C}$ for $10 \mathrm{~min}$ and stored at $5^{\circ} \mathrm{C}$ until experimentation. Gels were equilibrated at $25^{\circ} \mathrm{C}$ for $15 \mathrm{~min}$ prior to running NSE. Samples were aligned using a neutron camera, avoiding air bubbles and any other heterogeneities, and the scattering window was reduced to $3 \mathrm{~cm} \times 3 \mathrm{~cm}$. Two instrument wavelengths ( 8 and $11 \AA$ ) were used to capture a $q$ range of $0.36-1.34 \mathrm{~nm}^{-1}$ and a $t$ range (i.e., Fourier time) of 0.1-100 ns. Instrument resolution was measured using Grafoil ${ }^{\circledR}$ stacked graphite sheets (mid- and high- $q$ ) and aluminum oxide (low- $q$ ). Data at each position of the detector were reduced to get the normalized intermediate scattering function with solvent background correction by Eq. (1). Here, $A_{i}$ is the amplitude of the spin echo of species $i, T$ is the ratio of transmissions of the sample to the background, $U$ is the number of counts with no spin flip ("spin up," $\pi / 2$ and $\pi$ flippers off), and $D$ is the number of counts with a spin flip ("spin down," $\pi / 2$ flippers off, $\pi$ flipper on). In Eq. (1), there are three types of scatterers (species $i$ ): $P_{4}$ (sample), solvent background $(b)$, and resolution (res). Once $I(q, t)$ was obtained at each detector position, the data were binned and combined based on a set of $11 q$ arcs corresponding to different zones on the detector.

$I(q, t)=\left[\frac{2\left(A_{P 4}-T A_{b}\right)}{(U-D)_{P 4}-T\left(1-\phi_{P 4}\right)(U-D)_{b}}\right] /\left(\frac{2 A}{U-D}\right)_{\text {res }}$.

The intermediate scattering function $I(q, t)$ was fit to a Kohlrausch-Williams-Watts (KWW) stretched exponential function [Eq. (6)] [50]. Fitting was done with a weighted nonlinear least-squares algorithm in MATLAB using the objective function

$$
(\langle\tau\rangle, \beta)=\arg \min \sum\left(\frac{I(q, t)^{\text {expt }}-I(q, t)^{\mathrm{KWW}}}{d I(q, t)^{\mathrm{expt}}}\right)^{2} .
$$

The intermediate scattering function curves were also fit to the Zimm model [Eq. (7)] using nonlinear least-squares regression in MATLAB, where integrals were computed numerically using global adaptive quadrature [51].

Forced Rayleigh scattering $(F R S)$. FRS measurements were performed as previously described [36], using two different laser wavelengths $(\lambda=355$ and $488 \mathrm{~nm})$ to access diffusion length scales ranging from $150 \mathrm{~nm}$ to $50 \mu \mathrm{m}$. Each light source was a single-longitudinal mode laser at $100 \mathrm{~mW}$ (Coherent and Spectra-Physics for the 355- and 488-nm lasers, respectively). For self-diffusion measurements, $P_{4}$ hydrogels of various concentrations were prepared by hydrating proteins in $100 \mathrm{mM}$ sodium phosphate buffer at $p \mathrm{H} 7.6$ for 2 days, with $2 \%$ dye-labeled chains included in the gel. For tracer diffusion measurements, $P_{1}$-ONS or $P_{1}$-DTE was hydrated in a matrix of $P_{4}$ chains of the specified concentrations, with the tracer making up $\sim 1 \%$ of total coiled-coil stickers. This low $P_{1}$ concentration ensured that interaction between tracers was minimal. Gels were mixed periodically with a spatula or needle to ensure a homogeneous distribution of dye-labeled chains. ONS was used as the photochromic dye for measurements at $488 \mathrm{~nm}$, while DTE was used as the photochromic dye for measurements at $355 \mathrm{~nm}$. For measurements at 355 $\mathrm{nm}, 1 \mathrm{mM}$ 2-mercaptoethanol and $50 \mu \mathrm{M}$ ascorbic acid were also included in the gel as antioxidants to minimize spurious photoreactions. The presence of small-molecule antioxidants has been shown to minimally affect polymer diffusion in similar systems [47].

Samples for FRS were prepared by pressing the gels between two glass microscope slides separated by a $0.5-\mathrm{mm}$ Teflon spacer in an aluminum sample holder. Samples were annealed at $37^{\circ} \mathrm{C}$ for $2 \mathrm{~h}$ to allow the gels to fully relax, followed by equilibration at $25^{\circ} \mathrm{C}$ for $2 \mathrm{~h}$ before measurement. In the FRS measurements, two coherent laser beams of wavelength $\lambda$ were crossed onto the sample to selectively photoisomerize the dye-labeled chains, creating a holographic grating with period $d=\frac{\lambda}{2 n \sin (\theta / 2)}$, where $\theta$ is the angle at which the beams cross inside the gel and $n=1.35$ is the refractive index of the gel. The evolution of the resulting sinusoidal concentration profile was monitored by Bragg diffraction of one of the beams attenuated by $10^{4}-10^{5}$ to prevent further photoreactions in the sample. As in prior FRS experiments [36], the diffracted intensity was fit with a stretched exponential function

$$
I(t)=\left\{A \exp \left[-\left(\frac{t}{\tau}\right)^{\beta}\right]\right\}^{2}+B
$$

and the average diffusion time was computed as the first moment of the stretched exponential

$$
\langle\tau\rangle=\frac{\tau}{\beta} \Gamma\left(\frac{1}{\beta}\right),
$$

where $\Gamma$ is the Gamma function. The first $5-10 \mathrm{~s}$ of the decay curves were omitted from the fitting due to the presence of 
an additional fast time constant $(<3 \mathrm{~s})$ contributing to the initial decay. This fast time constant has been seen in prior experiments and is nondiffusive in nature [36]. A representative decay curve for self-diffusion of $P_{4}-\mathrm{ONS}$ at $6.5 \% \mathrm{w} / \mathrm{v}$ is shown in Fig. S12 [44].

\section{RESULTS AND DISCUSSION}

The model associative gel is formed by the artificial protein " $P_{4}$," which consists of four rodlike associating coiled-coil domains (" $P$ ") linked by flexible strands (" $C_{10}$ ") for a total molar mass of $62.8 \mathrm{kDa}$ [Fig. 1(a)]. In aqueous buffer, electrostatic and hydrophobic interactions between the $P$ domains result in their aggregation into pentameric bundles with orientational selectivity [18,52]. Above the overlap concentration of $\sim 5 \%$ $(\mathrm{w} / \mathrm{v})$, the protein forms an unentangled physical gel held by coiled-coil association [18,36,52] [Fig. 1(b)]. Previous studies using wide- and small-angle $\mathrm{x}$-ray scattering have shown that such coiled-coil bundles adopt a rigid cylindrical shape $\sim 1 \mathrm{~nm}$ in radius and several nanometers in length [52,53]. The specificity of coiled-coil association and the monodispersity of the connecting $C_{10}$ strands make the $P_{4}$ network ideal for studying relaxation and diffusion in a crowded gel environment formed by transient binding. Rheological characterization performed here (Fig. S5 [44]) and previously $[7,36]$ shows that $P_{4}$ hydrogels exhibit plateau moduli, relaxation, and terminal behavior typical of physically cross-linked networks [30].

Static structure. The static structure of a $P_{4}$ gel at $6.5 \%$ w/v and $25^{\circ} \mathrm{C}$ was probed with small-angle neutron scattering (SANS), revealing correlations spanning a range of length scales (Fig. 2). Quantitative estimates for characteristic structural features were obtained by fitting the intensity profile to a semiempirical broad-peak correlation length model [54]. Semiempirical correlation length models are commonly used to study polymer networks [55-62], as first-principles analytical expressions for network structure factors are available only for a very small number of specific chemically cross-linked systems. The broad-peak model used here has been shown to be applicable for gel networks containing clustered domains $[55,56,63,64]$ :

$$
I(q)=\frac{A}{q^{n}}+\frac{C}{1+\left(\left|q-q_{0}\right| \xi\right)^{m}}+B,
$$

where $q_{0}$ is the peak component, $\xi$ is a local correlation length, $n$ and $m$ are Porod and Lorentzian exponents, respectively, $A$ and $C$ are empirical parameters, and $B$ is the incoherent background. The first term in Eq. (5) describes low- $q$ clustering behavior, while the second term describes mid- to high- $q$ strand conformational behavior governed by polymer-solvent thermodynamics [63]. For the purposes of the fit, $I(q)$ was fit to the solvent background-subtracted scattering intensity, and $B$ was fixed prior to fitting, as detailed in the Supplemental Material [44]. The fit reveals a peak component $q_{0}=$ $0.36 \pm 0.04 \mathrm{~nm}^{-1}$, which can be used to identify a domain spacing of $d_{0}=18 \pm 2 \mathrm{~nm}$ (via $d_{0}=2 \pi / q_{0}$ ) corresponding to the average distance between coiled-coil bundles in the gel. This domain spacing is qualitatively consistent with, but slightly higher than, an estimate of $d_{\text {lattice }}=12.6 \mathrm{~nm}$ obtained by placing coiled-coil bundles on the vertices of a simple cubic lattice (Supplemental Material [44]). Given a free $C_{10}$

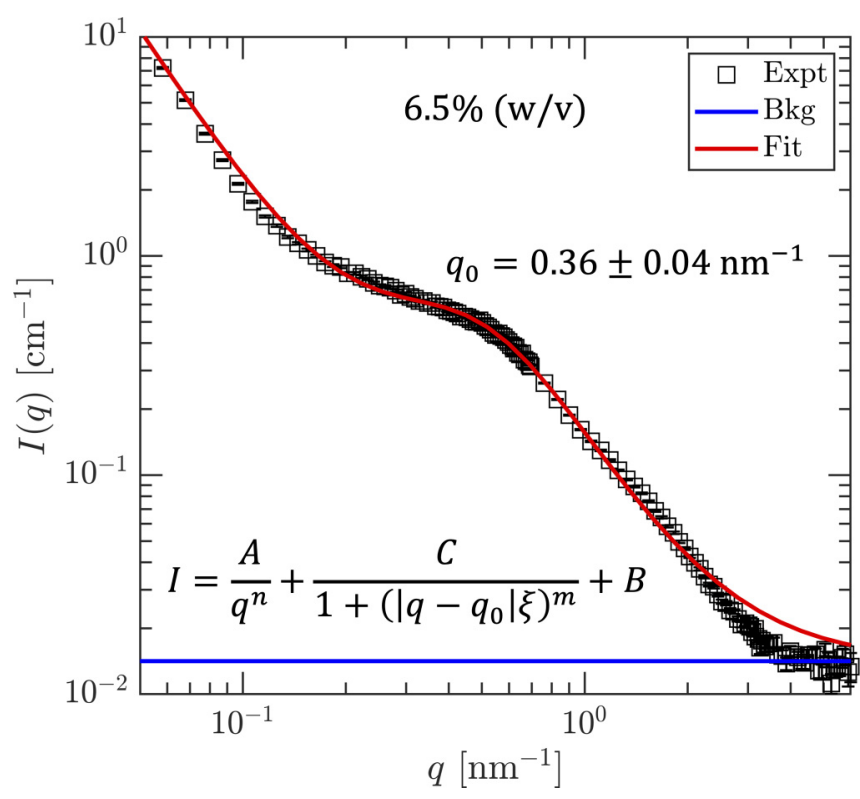

FIG. 2. Small-angle neutron scattering intensity curve for a $6.5 \%$ $P_{4}$ gel in deuterated buffer at $25^{\circ} \mathrm{C}$, fit to a broad-peak correlation length model (shown bottom left). Here, $q_{0}$ is the peak component wave vector, $\xi$ is a correlation length, $n$ and $m$ are Porod and Lorentzian exponents, respectively, and $A$ and $C$ are empirical parameters. The experimental scattering curve ("Expt") is the solvent background-subtracted scattering intensity. Thus, the background term shown here ("Bkg") arises only from incoherent scattering of the $P_{4}$ chains.

strand's equilibrium root-mean-square end-to-end distance of $6.6 \pm 0.7 \mathrm{~nm}$ in aqueous buffer [65], there may be mild stretching of bridging chains and/or clustering of multiple pentameric bundles, resulting in the larger junction spacing measured by SANS. The local correlation length is found to be $\xi=2.6 \pm 0.2 \mathrm{~nm}$, providing a measure of the blob size of the $C_{10}$ strands, beyond which excluded volume interactions are screened and chains exhibit Gaussian conformational statistics $[57,61,62,66]$. The Lorentzian exponent $m=1.95 \pm 0.1$ indicates that local strand conformations are between those in $\theta$ - and good-solvent conditions [57], consistent with prior dynamic light scattering measurements of the $C_{10}$ domain in aqueous buffer [65]. In the low- $q$ region, the SANS intensity exhibits an upturn for $q<0.11 \mathrm{~nm}^{-1}$ with a Porod scaling of $q^{-2.39 \pm 0.1}$, indicating partial higher-order clustering on length scales $>57 \mathrm{~nm}$ [54]. While the characteristic cluster size cannot be determined within the $q$ range of the SANS instrument, all gels are optically clear, indicating the absence of macroscopic clusters, inhomogeneities, or phase separation. Finally, the high- $q$ scattered intensity follows a scaling of $I(q) \sim q^{-4.6 \pm 0.5}$, consistent with Porod's law of $I(q) \sim q^{-4}$ expected for smooth fractal objects [54,60], which here may arise from the smooth surface of the coiled-coil bundles.

Segmental motion. Segmental relaxation of $P_{4}$ chains within the gel was measured using neutron spin-echo (NSE) spectroscopy, which accesses dynamics on submolecular to molecular length scales. The measurement range of $q=$ $0.36-1.34 \mathrm{~nm}^{-1}$ was chosen such that the NSE signal resulted predominantly from coherent scattering of the $P_{4}$ chains 

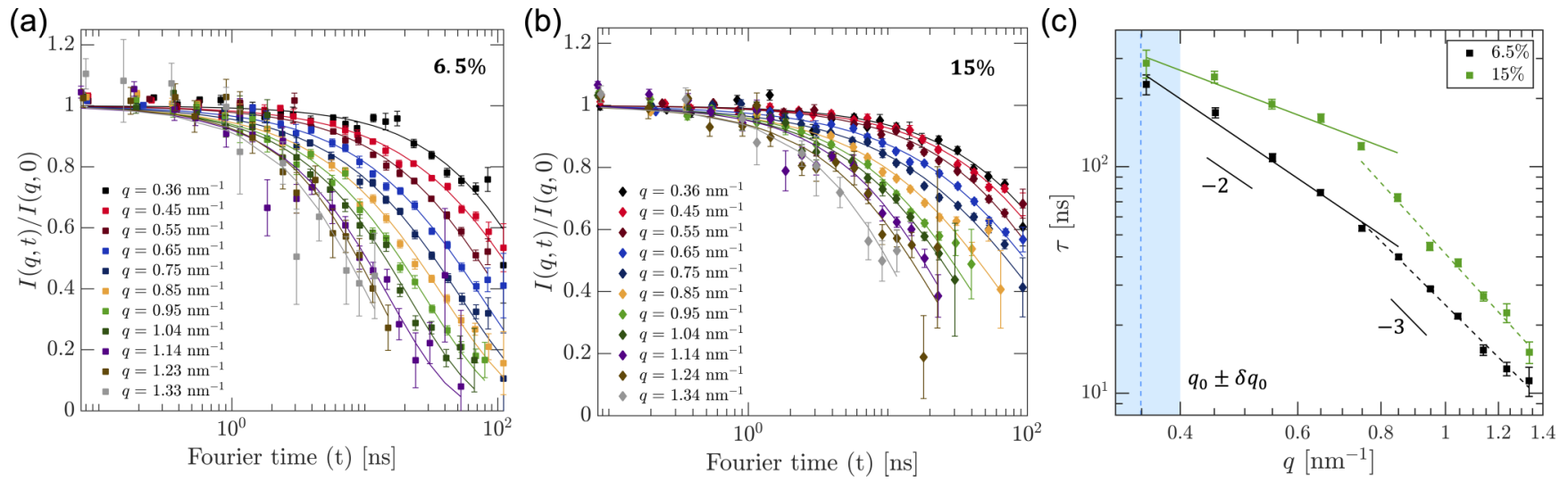

FIG. 3. Normalized coherent intermediate scattering function measured by neutron spin-echo spectroscopy for $P_{4}$ gels at $25^{\circ} \mathrm{C}$ at concentrations of (a) $6.5 \%$ and (b) $15 \%$ w/v. Solid lines are fits to a phenomenological Kohlrausch-Williams-Watts (KWW) streteched exponential function [Eq. (6)]. (c) Relaxation time $\tau$ as a function of wave vector $q$ for each gel. Error bars represent 1 standard deviation from 100 bootstrapped replicates of the intermediate scattering function, which were each fit to the KWW function. Lines are power-law fits for each scaling regime. The black solid labels show the expected scaling for Zimm dynamics $\left(\tau \sim q^{-3}\right)$ and Fickian diffusion $\left(\tau \sim q^{-2}\right)$. The blue shaded region shows the peak component wave vector and its $95 \%$ confidence region, $q_{0} \pm \delta q$, obtained from SANS.

(see Figs. 2 and S7 [44]), thus providing a description of the dynamic pair-correlation function of the various chain segments, including both the coiled-coil bundles and flexible linker strands. The real-space length scale range accessed by NSE of $d=5-18 \mathrm{~nm}$ (via $d=2 \pi / q$ ) overlapped with the $P_{4}$ radius of gyration $\left(R_{g} \approx 17 \mathrm{~nm} \mathrm{[36])} \mathrm{and} \mathrm{network} \mathrm{junction}\right.$ spacing ( $d_{0}=18 \pm 2 \mathrm{~nm}$ ) estimated from SANS. Figures 3(a) and 3 (b) show normalized dynamic coherent scattering curves at various wave vectors measured by NSE for $P_{4}$ gels at concentrations of $6.5 \%$ and $15 \%(\mathrm{w} / \mathrm{v})$ at $25^{\circ} \mathrm{C}$. Due to the complex molecular structure of the $P_{4}$ chains, the dynamic scattering curves were fit to a phenomenological KohlrauschWilliams-Watts (KWW) stretched exponential $[67,68]$ of the form

$$
\frac{I(q, t)}{I(q, 0)}=\exp \left[-\left(\frac{t}{\tau}\right)^{\beta}\right]
$$

yielding the relaxation time $\tau$ and line-shape parameter $\beta$ corresponding to the wave vector $q$ [69]. As seen in Figs. 3(a) and 3 (b), for all cases the intermediate scattering function is well captured by Eq. (6). The line-shape parameter $\beta$ ranges from 0.7 to 1 for all curves (Fig. S8 in the Supplemental Material [44]), suggesting mild heterogeneity in the relaxation spectrum [67]. While complete decay of $I(q, t)$ is not observed within the Fourier time range of the NSE instrument, the curves do not show signs of a plateau that would be expected for "frozen" fluctuations within the gel, suggesting mobility of both the coiled-coil bundles and the linker strands within the investigated $q$ range.

Two power-law scaling regimes are observed for both gel concentrations, with a transition around $q_{c} \approx 0.75 \mathrm{~nm}^{-1}$, as shown in Fig. 3(c). For $q>q_{c}$, the $6.5 \%$ gel shows a scaling of $\tau \sim q^{-2.9 \pm 0.4}$ while the $15 \%$ gel shows a scaling of $\tau \sim q^{-3.2 \pm 0.6}$, where uncertainties indicate $95 \%$ confidence intervals. These scaling exponents are consistent with Zimm dynamics $\left(\tau \sim q^{-3}\right)$, suggesting that despite the complex molecular structure of the $P_{4}$ chain, local dynamics resemble the segmental modes of a free chain in solution. Here, this behavior is likely dominated by fluctuations of the flexible
$C_{10}$ linker strands on length scales smaller than the spacing between coiled-coil junctions. For $q<q_{c}$, the $6.5 \%$ gel transitions to a scaling of $\tau \sim q^{-2.0 \pm 0.5}$, consistent with Fickian diffusion $\left(\tau \sim q^{-2}\right)$. This diffusive regime may arise from collective segmental diffusion $[68,70]$ and/or overdamped gel modes involving concentration fluctuations on length scales greater than the network mesh size, governed by the balance between network elasticity and polymer-solvent friction $[71,72]$. In contrast, the $15 \%$ gel transitions to a surprisingly shallow scaling of $\tau \sim q^{-1.1 \pm 0.4}$, which may arise from an enhancement of multichain correlations at this higher concentration [66]; however, its origin remains uncertain. The onset of Fickian diffusion occurs at a real-space length scale of $d_{c}=$ $2 \pi / q_{c} \approx 8.4 \mathrm{~nm}$, which is likely related to the network junction spacing [72], providing another estimate of the distance between coiled-coil bundles comparable to that from SANS. In addition, the transition time scale $\tau_{c}$ can be interpreted as the approximate Zimm time of the $C_{10}$ domain in each gel, which is $\tau_{c} \approx 60 \mathrm{~ns}(6.5 \%)$ and $120 \mathrm{~ns}(15 \%)$. Within both regimes, the $15 \%$ gel's relaxation times are only 1-3 times those of the $6.5 \%$ gel, despite their long-time chain diffusivities being orders of magnitude apart. Thus, while sticker association/dissociation dynamics control macroscopic network dynamics, junctions can be considered permanently bound and chain conformational relaxation largely decoupled from sticker dynamics on the short time scales accessed by NSE [72].

To further probe the Zimm-like behavior of the $P_{4}$ gels at high $q$, the normalized intermediate scattering functions for each gel were examined as a function of the reduced Zimm time $\left(q^{3} t\right)^{2 / 3}$, which is the natural scaling variable of the coherent intermediate scattering function in the Zimm model [69]. In the Zimm model, the coherent intermediate scattering function in the limit of an infinitely long chain in $\theta$ conditions is given by [69]

$$
\frac{I(q, t)}{I(q, 0)}=\int_{0}^{\infty} d u \exp \left(-u-\left(\Gamma_{z} t\right)^{2 / 3} h\left(u\left(\Gamma_{z} t\right)^{-2 / 3}\right)\right),
$$



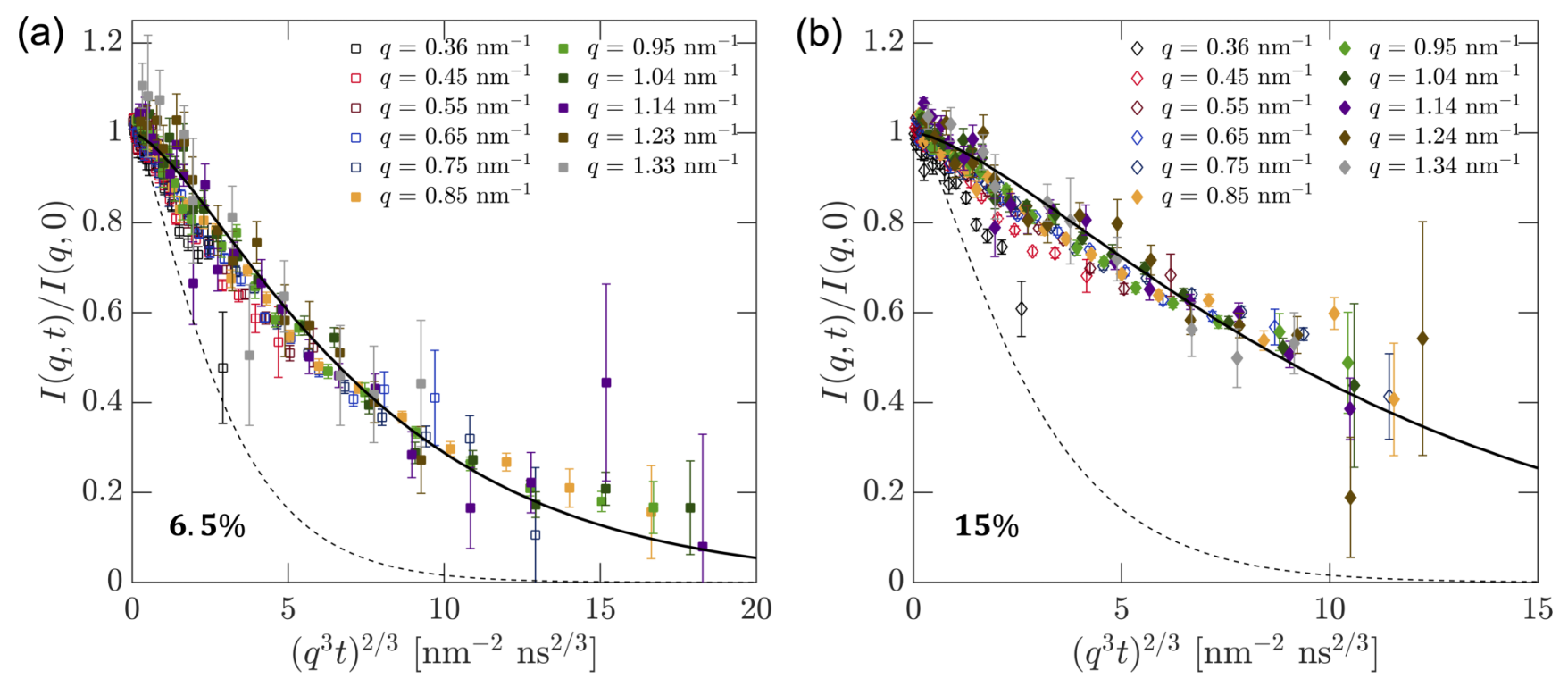

FIG. 4. Zimm scaling of the normalized intermediate scattering functions for the (a) $6.5 \%$ and (b) $15 \%$ gels. Closed symbols are used for curves in the Zimm-like regime $\left(q>0.75 \mathrm{~nm}^{-1}\right)$ and open symbols are used for curves in the Fickian diffusion regime $\left(q \leqslant 0.75 \mathrm{~nm}^{-1}\right)$, with the transition wave vector of $q_{c}=0.75 \mathrm{~nm}^{-1}$ determined from the $\tau$ vs $q$ scaling regimes shown in Fig. 3(c). The solid black curves are least-squares fits to the Zimm model [Eq. (7)] of the curves for $q>0.75 \mathrm{~nm}^{-1}$, treating $\Gamma_{z}$ as a fitting parameter. The decay rates resulting from the fits are $\Gamma_{z} / q^{3}=0.055 \pm 0.003$ and $0.033 \pm 0.002 \mathrm{~nm}^{3} \mathrm{~ns}^{-1}$ for the $6.5 \%$ and $15 \%$ gels, respectively. The dashed curves are the predicted intermediate scattering function for an ideal single chain using the decay rate $\Gamma_{z, \theta}$ given by Eq. (7c).

where

$$
h(y)=\frac{2}{\pi} \int_{0}^{\infty} d x \frac{\cos (x y)}{x^{2}}\left[1-\exp \left(-\frac{x^{3 / 2}}{\sqrt{2}}\right)\right]
$$

and $\Gamma_{z}$ is the Zimm decay rate, predicted in $\theta$ conditions to be

$$
\Gamma_{z, \theta}=\frac{k_{B} T}{6 \pi \eta_{s}} q^{3},
$$

where $\eta_{s}$ is the solvent viscosity $\left(9 \times 10^{-4} \mathrm{~Pa}\right.$ s for the $0.1 \mathrm{M}$ sodium phosphate buffer used here), $k_{B}$ is Boltzmann's constant, and $T$ is temperature $\left(25^{\circ} \mathrm{C}\right)$. In this work, $\Gamma_{z}$ is treated as a fitting parameter to account for deviations from $\theta$ conditions and the high chain concentration (above overlap) in the gels. For each gel concentration, the Zimm decay rate $\Gamma_{z}$ was determined by simultaneously fitting the Zimm-scaled dynamic scattering curves for $q>0.75 \mathrm{~nm}^{-1}$ [corresponding to the Zimm-like regime shown in Fig. 3(c)] to Eq. (7) using nonlinear least-squares regression. As shown in Fig. 4, for each concentration, the dynamic scattering curves at $q>$ $0.75 \mathrm{~nm}^{-1}$ largely collapse onto a master curve when plotted against the reduced Zimm time, in reasonable, but not perfect, agreement with the Zimm model (solid black curves). However, the curves at $q \leqslant 0.75 \mathrm{~nm}^{-1}$ appear to decay more steeply than the Zimm model due to the transition to Fickian diffusion, consistent with the scaling regimes shown in Fig. 3(c). The decay rates (normalized by $q^{3}$ ) obtained from the fits are $\Gamma_{z} / q^{3}=0.055 \pm 0.003 \mathrm{~nm}^{3} \mathrm{~ns}^{-1}$ for the $6.5 \%$ gel and $0.033 \pm 0.002 \mathrm{~nm}^{3} \mathrm{~ns}^{-1}$ for the $15 \%$ gel, where uncertainties are $95 \%$ confidence intervals. The characteristic times given by $\Gamma_{z}^{-1}$ are within a factor of 1.4 of the time constants $\tau$ obtained from the KWW fits [Eq. (6)] of the NSE data in the high- $q$ regime, as shown in Fig. S11 [44], demonstrating qualitative agreement. It is notable that the values of $\Gamma_{z}$ for the $6.5 \%$ and $15 \%$ gels are 4.4 and 7.3 times smaller, respectively, than the predicted decay rate of Eq. (7c) for an ideal single chain (dashed curves in Fig. 4). In addition, even at high $q$, the collapsed dynamic scattering curves for both concentrations show qualitative deviations from the prediction of Eq. (7) (which is rigorous in the limit of an infinitely long chain in dilute $\theta$ conditions [69]). The slower decay rates in the $P_{4}$ gels and the deviations from the ideal Zimm limit, even on local length scales, may be attributed to contributions from the slower dynamics of the rigid coiled-coil bundles, the relatively short length of the $C_{10}$ linkers ( $<100$ Kuhn segments), strand tethering due to binding, and an increase in the effective local viscosity due to crowding of the overlapping strands (effects expected to be more pronounced in the $15 \%$ gel due to its higher concentration) $[69,73]$. However, for both concentrations, the dynamic scattering curves at $q>0.75 \mathrm{~nm}^{-1}$ appear well parametrized by the scaling of $\left(q^{3} t\right)^{2 / 3}$, supporting the Zimm-like segmental behavior suggested by the $\tau \sim q^{-3}$ scaling in Fig. 3(c).

Self-diffusion. Center-of-mass self-diffusion of the $P_{4}$ chains on length scales greater than their radius of gyration was next probed by forced Rayleigh scattering (FRS), which accesses diffusion on supramolecular length scales up to the terminal Fickian regime. FRS measurements were performed as previously described [36], with $\sim 2 \%$ of chains labeled with a photochromic dye. The use of two laser wavelengths (355 and $488 \mathrm{~nm}$ ) enabled a wide experimental range of $150 \mathrm{~nm}<$ $d<50 \mu \mathrm{m}$, corresponding to $\sim 10 R_{g}$ up to $\sim 3000 R_{g}$. Briefly, two laser beams of wavelength $\lambda$ were crossed in the sample at an angle $\theta$, selectively photoisomerizing the dye-labeled chains to create a sinusoidal concentration profile with period $d=\frac{\lambda}{2 n \sin (\theta / 2)}$, where $n=1.35$ is the gel refractive index. 
(a)

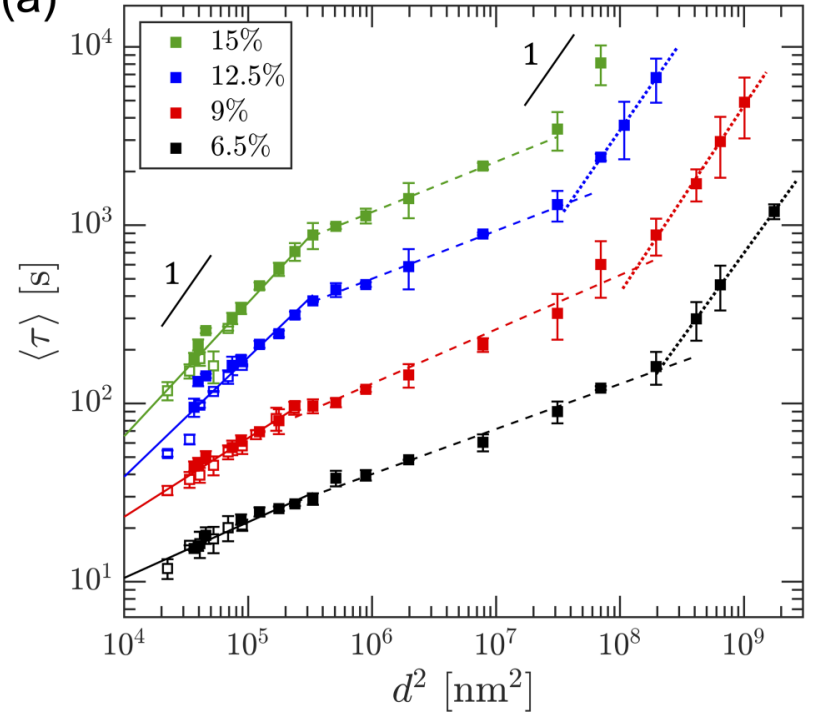

(b)

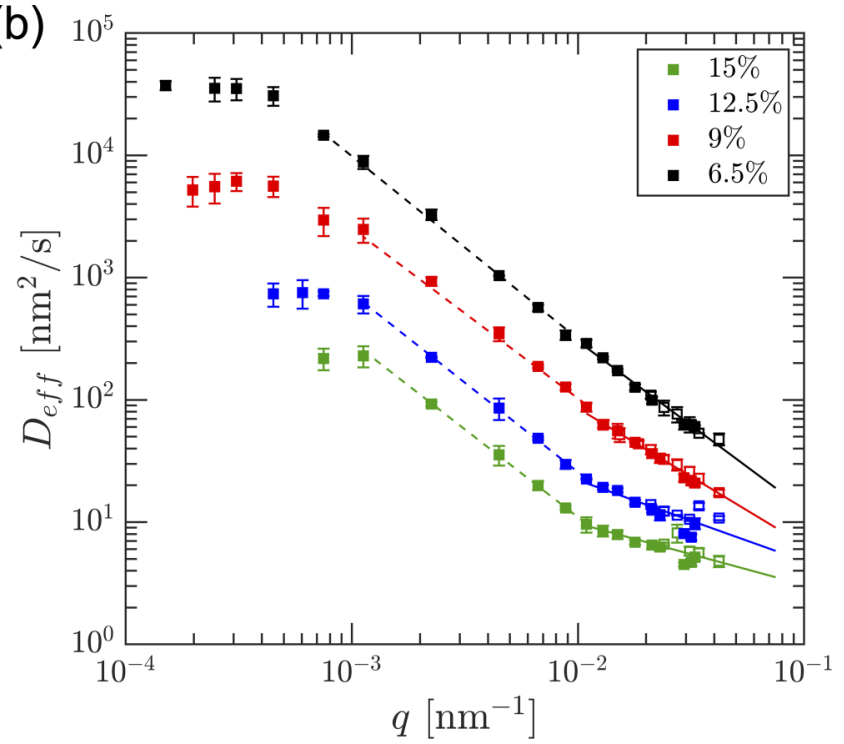

FIG. 5. (a) Forced Rayleigh scattering measurements of mean self-diffusion time $\langle\tau\rangle$ as a function of length scale squared $d^{2}$ in $P_{4}$ gels of various concentrations at $25^{\circ} \mathrm{C}$, showing two sequential regimes of apparent superdiffusive scaling (slope less than 1) followed by terminal Fickian diffusion (slope of 1). Lines are power-law fits to $\langle\tau\rangle \sim d^{2 \mu}$ for each regime. (b) Effective self-diffusion coefficient $D_{\text {eff }}=d^{2} / 4 \pi^{2}\langle\tau\rangle$ as a function of the wave vector $q=2 \pi / d$, showing the transitions in $D_{\text {eff }}$ in each regime. Lines are power-law fits to each regime. In both panels, open symbols are data collected at $355 \mathrm{~nm}$, and closed symbols are data collected at $488 \mathrm{~nm}$.

The evolution of this concentration profile was monitored by Bragg diffraction of one of the beams to determine the diffusion time scale $\tau$ corresponding to the length scale $d$. Because the photoisomerized, native, and unlabeled chains are all approximately identical, there is minimal perturbation of the system away from equilibrium, and the relaxation rate may be assumed equal to the equilibrium self-diffusion rate within the gel [74].

Figure 5(a) shows mean self-diffusion times $\langle\tau\rangle$ for various values of the sinusoidal period $d$ for $P_{4}$ gels of various concentrations at $25^{\circ} \mathrm{C}$. For all cases, the diffracted intensity decayed as a single stretched exponential with mean time constant $\langle\tau\rangle$ (details of the fitting procedure are in the Experiment section). $P_{4}$ diffusion shows three distinct scaling regimes with increasing $d^{2}$, described by the power-law relationship $\langle\tau\rangle \sim d^{2 \mu}$ where $\mu=1$ for Fickian diffusion. Chains first undergo two distinct regimes of apparent superdiffusion $(\mu<$ 1) on intermediate length scales, with a transition around $d^{2} \approx 2.5 \times 10^{5} \mathrm{~nm}^{2}$, followed by terminal Fickian diffusion on large length scales. While previous experiments $[36,37]$ have observed the upper, more extreme superdiffusive regime $\left(d^{2}>2.5 \times 10^{5} \mathrm{~nm}^{2}\right)$, this observation of a distinct lower superdiffusive regime suggests a transition to a different set of diffusive modes on these smaller length scales $(\sim 10-30$ times the chain radius of gyration). Unlike in the upper superdiffusive regime, the power-law exponents in the lower superdiffusive regime exhibit a strong concentration dependence, with $\mu=0.31 \pm 0.05,0.44 \pm 0.04$, $0.67 \pm 0.1$, and $0.75 \pm 0.1$ for the $6.5,9,12.5$, and $15 \%$ $(\mathrm{w} / \mathrm{v})$ gels, respectively. This increase in the power-law exponent of the lower superdiffusive regime (i.e., closer to Fickian dynamics) correlates with a decrease in the width of the upper superdiffusive regime, indicating a reduction in the overall extent of superdiffusive scaling at higher concentration.

The effective self-diffusion coefficient $D_{\text {eff }}$ at each $d$ spacing (or inverse wave vector $q$ ) can be determined from the FRS decay time via

$$
D_{\text {eff }}=\frac{d^{2}}{4 \pi^{2}\langle\tau\rangle}=\frac{1}{q^{2}\langle\tau\rangle}
$$

where for Fickian diffusion $D_{\text {eff }}$ is independent of $q$. The FRS data demonstrate that $D_{\text {eff }}$ for the $P_{4}$ chains is highly $q$ dependent and decreases with concentration [Fig. 5(b)]. At low $q, D_{\text {eff }}$ is constant for all concentrations, corresponding to the terminal Fickian regime at large $d^{2}$. The terminal diffusivity shows a strong inverse dependence on concentration $(\phi)$ with a scaling of $D_{\text {eff }} \sim \phi^{-6.1 \pm 0.5}$ (Fig. S13 [44]), which can be attributed to an increased density of binding sites within the network that hinders chain mobility. This concentration dependence is qualitatively consistent with, but slightly stronger than, the scaling prediction of the sticky Rouse model of $D_{\text {eff }} \sim \phi^{-5.19}$ for good solvent conditions in this concentration regime [31]. The deviation from the scaling prediction has been observed in prior measurements of coiledcoil protein diffusion [36] and may arise from the pentameric nature of coiled-coil aggregation, compared to the pairwise association assumed in the sticky Rouse model. At higher $q, D_{\text {eff }}$ decreases with $q$ for all concentrations, displaying two power-law scaling regimes that correspond to the two superdiffusive regimes seen at low $d^{2}$ in Fig. 5(a). Notably, the high- $q$ scaling of $D_{\text {eff }}$ suggests a convergence to a common effective diffusivity with further increases in $q$, beyond the range of FRS, suggestive of a limiting intrinsic relaxation time scale independent of concentration on small length scales (vide infra). 
The regimes of apparent superdiffusion seen in Fig. 5 may be attributed to transitions between the relative contributions of different diffusive modes within the network (e.g., hopping, walking, or multichain cluster motion), each over a finite length scale range. While previous analyses [36,37] have attempted to describe associative polymer diffusion using two-state models, where superdiffusive scaling arises due to an interconversion from a bound ("slow") state to a hopping ("fast") state upon unbinding from the network, restricting bound-state diffusion to one dynamic mode fails to capture the multiple superdiffusive regimes observed here, instead predicting purely Fickian diffusion on small $d$ spacings [36]. A transition to a hopping mode has also been invoked in a recent Brownian dynamics simulation study of associating star polymers [34] to explain apparent superdiffusive behavior, corresponding approximately to the upper superdiffusive regime observed here. The presence of two distinct superdiffusive regimes in Fig. 5(a), however, suggests that the transitions between diffusive states other than hopping may also result in apparent superdiffusion on smaller length scales (though still $>$ tenfold the chain radius of gyration). The FRS results thus provide evidence for a complex ensemble of more than two molecular states within the gel, where transitions between different sets of states can result in multiple regimes of apparent superdiffusion on different length scales. This ensemble of molecular configurations is also expected in view of the four stickers per chain on the $P_{4}$ molecule, which allow for substantially more than two states compared to architecturally simpler networks containing only one or two stickers per chain $[33,75]$. While some aspects of the self-diffusive behavior here resemble a Lévy flight process (where molecules undergo a heavy-tailed distribution of jump lengths, resulting in an increase in the effective diffusivity over time [76,77]), it is important to note that each diffusive state (walking, hopping, etc.) is expected to be an equilibrium random walk that results in a Gaussian spatial distribution of its respective population [34]. The difference in each population's diffusivity and changes in the relative contribution of each state toward the overall chain diffusivity are the proposed origin of the regimes of apparent superdiffusive scaling observed here.

At higher concentrations, the reduced prominence of both superdiffusive regimes suggests a decrease in the average hopping displacement and frequency and an overall convergence in the contributions of the various diffusive modes in the gel. The suppression of hopping may originate from an increase in binding site density, changes in network topology (e.g., fewer loops), and an increase in the effective bond lifetime due to repeated sticker dissociation and reassociation events before partner exchange $[31,36,78]$. However, from the FRS data alone it is difficult to estimate the characteristic displacement of a walking or hopping step in the $P_{4}$ network due to the inability to decouple the relative contributions of the various diffusive mechanisms to network relaxation. Previous studies on proteins containing two stickers per chain have estimated a root-mean-squared hopping displacement of $\sim 1.1 \mu \mathrm{m}$, where hopping was assumed to be the only diffusive mode due to the small number of stickers per chain [33]. The $P_{4}$ characteristic hopping displacement may be similar to this value, although it is expected to be lower due to its increased number of stickers per chain and greater contribution from other modes.
As shown in Fig. 5, $P_{4}$ chains ultimately transition from the upper superdiffusive regime to terminal Fickian scaling at length scales $10^{3}-10^{4}$ times the chain radius of gyration, depending on the concentration, where the effective diffusivity is expected to contain contributions from hopping, walking, and cluster diffusion to varying degrees [34,36].

The combination of the NSE and FRS data provides insight into the relationship between submolecular relaxation and sticker-exchange-mediated diffusion in the associative network. In Fig. 6(a) the NSE and FRS relaxation times are plotted together against the real-space length scale squared, where length and time are normalized by the lattice-model estimate of the coiled-coil junction spacing $\left(d_{\text {lattice }}=12.6 \mathrm{~nm}\right)$ and $C_{10}$ Zimm time approximated from NSE measurements, respectively. (The same data in non-normalized units are presented in Fig. S14 [44].) Extrapolating the relaxation times from NSE up to the larger length-scale times from FRS reveals the presence of one or more caging regimes in the intermediate region between $0.06<d^{2} / 4 \pi^{2} d_{\text {lattice }}^{2}<3.6$ (equivalently, $20 \mathrm{~nm}<d<150 \mathrm{~nm}$ ). These caging regimes likely correspond to structural features within the gel, as is widely seen in diffusion in heterogeneous systems, particularly biological media $[1,6,9,13-15]$.

Comparing the experimental data to recent Brownian dynamics simulations on associating star polymers [34] allows estimation of the time and length scales governing the caging regime in the $P_{4}$ gel. As seen in Figs. 6(a) and 6(b), several aspects of the experimental results show qualitative agreement with simulation, including fast submolecular relaxation, caging, multiple apparent superdiffusive regimes that are more pronounced at lower concentration, and terminal Fickian diffusion. Although in the simulations the extent of superdiffusive scaling decreases with concentration until it is no longer present around $\phi / \phi^{*}=10$, the maximum concentration probed experimentally is $\phi / \phi^{*} \approx 3$ due to limitations in the time scales accessible by FRS. It is expected that further increasing the concentration would decrease the width of the regime over which superdiffusive scaling is observed, though complete disappearance may be experimentally inaccessible due to the onset of chain entanglement at higher concentrations.

Simulations [Fig. 6(b)] predict a caging regime on length scales similar to the root-mean-square extension of the strands [34]. Diffusion over larger distances requires one or more stickers to unbind, decreasing chain mobility by a degree determined by the sticker dissociation kinetics. The normalized FRS curves can be extrapolated down to shorter length scales where they appear to intersect around $d^{2} / 4 \pi^{2} d_{\text {lattice }}^{2} \approx$ $0.1-0.4$ and $\tau / \tau_{\text {Zimm }} \approx 10^{8}$, or in real units, $d^{*} \approx 25-50 \mathrm{~nm}$ and $\tau^{*} \approx 6-12 \mathrm{~s}$. These are predicted to be characteristic length and time scales corresponding to a molecule's exit from the cage, independent of concentration, signifying its transition from submolecular relaxation to larger-scale diffusion mediated by sticker exchange. In particular, the time scale $\tau^{*}$ is proposed to originate from an intrinsic, concentrationinvariant sticker dissociation time [34]. On greater time scales, multiple association/dissociation events can occur, leading to different diffusive modes and concentration-dependent dynamics. As seen in Fig. 6(a), the dissociation time $\tau^{*}$ estimated from FRS is qualitatively consistent with the coiled-coil 

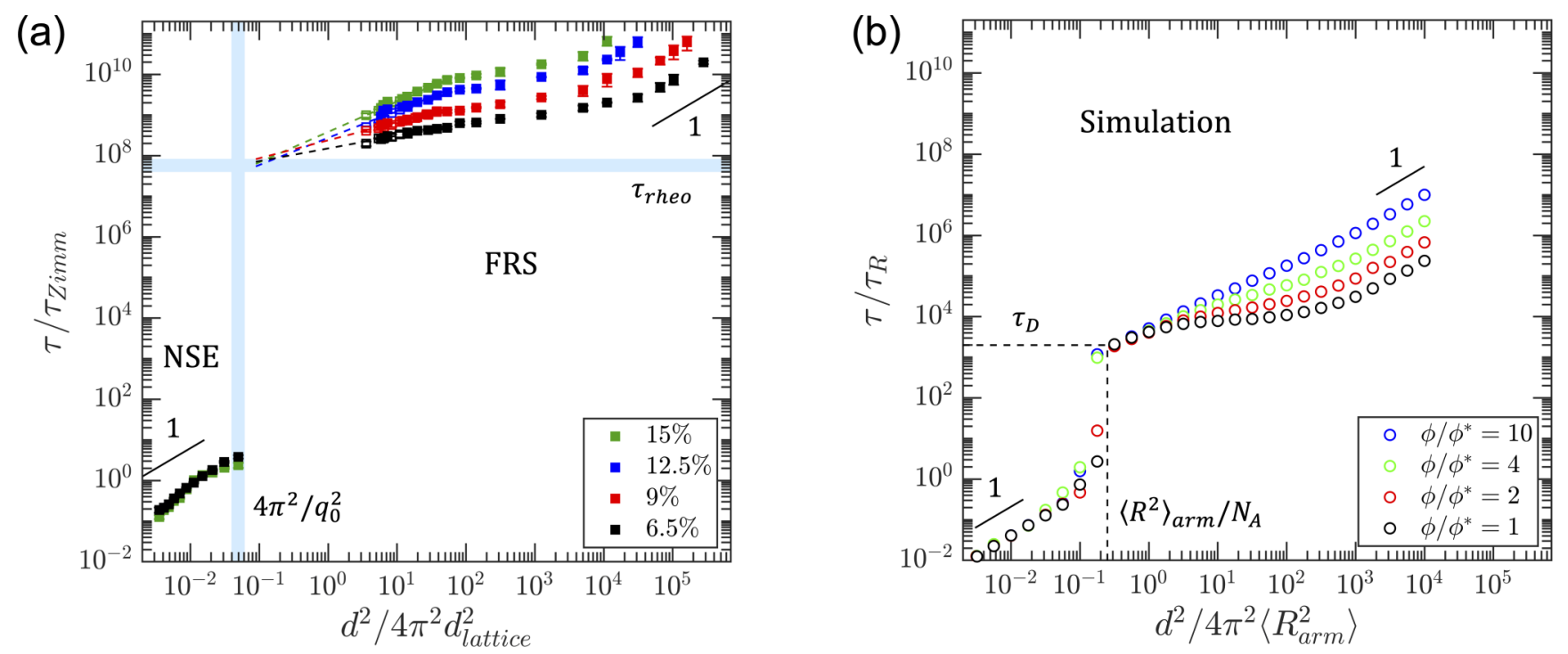

FIG. 6. (a) Combined NSE and FRS results for $P_{4}$ hydrogels of different concentrations at $25^{\circ} \mathrm{C}$. Length and time are normalized by the coiled-coil junction spacing and the $C_{10}$ Zimm time, respectively. Dashed lines are an extrapolation to a hypothesized intrinsic, concentrationindependent sticker dissociation time. The vertical shaded region indicates the length scale corresponding to the coiled-coil junction spacing $95 \%$ confidence region obtained from SANS $\left(q_{0}=0.36 \pm 0.04 \mathrm{~nm}^{-1}\right)$. The horizontal shaded region indicates the range of network relaxation times measured by rheology for the gel concentrations probed here $\left(2.4 \mathrm{~s}<\tau_{\text {rheo }}<10.1 \mathrm{~s}\right.$, obtained from Ref. [36]). (b) Characteristic time as a function of domain length squared from Brownian dynamics simulations of associating four-arm star polymers, based on data from Ref. [34]. Concentration is normalized by the overlap concentration $\phi^{*}$. Length and time are normalized by the mean-square arm length $\left\langle R^{2}\right\rangle_{\text {arm }}$, and arm relaxation time $\tau_{R}$, respectively. The characteristic length and time governing the subdiffusive cage are indicated as $\left\langle R^{2}\right\rangle_{\text {arm }} / N_{A}\left(\right.$ where $N_{A}=4$ is the number of arms per molecule) and the sticker dissociation time $\tau_{D}$, respectively.

exchange time measured by rheology $\left(2.4 \mathrm{~s}<\tau_{\text {rheo }}<10.1 \mathrm{~s}\right.$, horizontal shaded region; Fig. S5 [44] and Ref. [36]). The dimensionless cage size $d^{*}$ is also similar to the spacing between coiled-coil bundles measured by SANS $\left(d_{0}=18 \pm 2\right.$ $\mathrm{nm}$, vertical shaded region), in qualitative agreement with simulation. However, caging is not directly observed in the NSE data (Fig. 3). Cooperative junction motion may increase the effective cage size to outside the NSE $q$ range; this diffusive mode was neglected in the simulations [34] and may be a source of error in the estimates of $\tau^{*}$ and $d^{*}$. Overall, the experimental results qualitatively support many predictions from simulation and allow estimation of molecular parameters (e.g., junction spacing and binding kinetics) governing the caging regime in the $P_{4}$ gel. However, since the simulations in Ref. [34] only track center-of-mass motion, they do not capture the segmental relaxation behavior measured by NSE, instead predicting purely Fickian diffusion on submolecular length scales.

Single-sticker tracer diffusion. To more directly probe individual sticker binding dynamics within the $P_{4}$ gel, tracer diffusion of a single-sticker protein analog (" $P_{1}$ ") through the $P_{4}$ gels was measured using FRS. $P_{1}$ contains a single $P$ domain flanked by two $C_{5}$ strands (the same repeating sequence as $C_{10}$ except half the length), giving it the structure $C_{5} P C_{5}$ with a molar mass of $16.6 \mathrm{kDa}$ [see Fig. 1(a)]. This structure was chosen to resemble the local chemical structure of a single coiled-coil domain in the $P_{4}$ network, including steric effects from the flanking linker chains. For tracer diffusion measurements, a low concentration of $P_{1}$ ( $\sim 1 \%$ of all coiled-coil domains) was dispersed into the $P_{4}$ gel such that interaction between $P_{1}$ tracers could be assumed negligible. Diffusion of $P_{1}$ through the $P_{4}$ matrix was expected to be controlled by its rates of binding and unbinding from the network, enabling direct measurement of the kinetics of association of the coiledcoil domains in the network. In particular, the strictly two molecular configurations of the $P_{1}$ molecule (i.e., free and bound) enable rigorous analysis using a two-state model, as in prior analysis of small-molecule sticker diffusion through metal-coordinating gels [75].

Figure 7 shows tracer diffusion measurements of $P_{1}$ through $P_{4}$ gels, along with a comparison to $P_{4}$ self-diffusion measurements at the same concentrations. Both the tracer and self-diffusive behavior are qualitatively similar, exhibiting superdiffusive scaling on intermediate length scales followed by Fickian scaling on long length scales. Although $P_{1}$ tracer diffusion is faster than $P_{4}$ self-diffusion for each gel concentration, the tracer and self-diffusion times are within a factor of 3 for the $6.5 \%$ gel and within a factor of 2 for the $15 \%$ gel, a surprising degree of similarity considering the difference in the number of stickers per chain (4 vs 1).

To estimate the coiled-coil dissociation time within the gel, the tracer diffusion data were fit to a previously developed two-state reaction-diffusion model that captures the effect of transient association on diffusion through the gel $[36,75]$. Briefly, the model postulates that $P_{1}$ tracers diffuse through the network via two states, either free (denoted $P_{1}^{F}$ ) or bound to the network (denoted $P_{1}^{B}$ ). Each state has its own characteristic diffusivity $\left(D_{F}\right.$ and $\left.D_{B}\right)$, where the bound-state diffusivity can be nonzero due to collective rearrangement of the network structure as a whole. Interconversion between the states occurs 


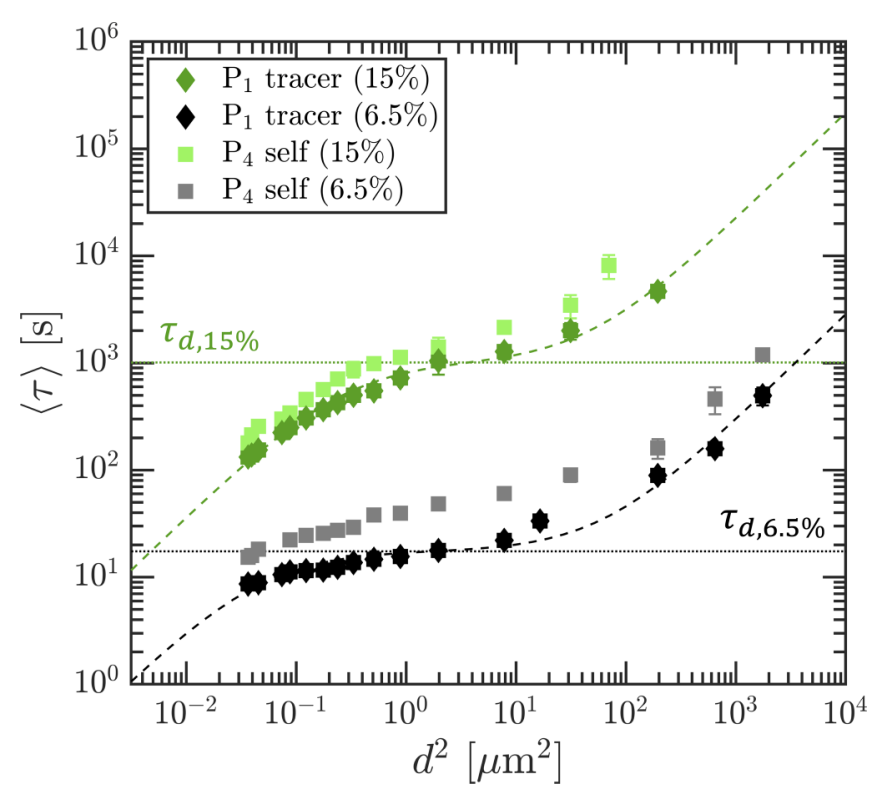

FIG. 7. Forced Rayleigh scattering measurements of tracer diffusion of single-sticker $P_{1}$ chains in a matrix of $P_{4}$, compared to $P_{4}$ self-diffusion. Dashed lines are fits of the $P_{1}$ diffusion data to the two-state model, as described in the text. The horizontal dotted lines show the characteristic $P_{1}$ dissociation time in each gel, given by $\tau_{d}=k_{d}^{-1}$.

by first-order chemical kinetics:

$$
P_{1}^{F} \underset{k_{d}}{\stackrel{k_{a}}{\rightleftharpoons}} P_{1}^{B},
$$

where $k_{a}$ and $k_{d}$ are the association and dissociation rate constants, respectively. A material balance yields the following system of equations governing the diffusion of each species and interconversion between the two states:

$$
\begin{gathered}
\frac{\partial\left[P_{1}^{F}\right]}{\partial t}=D_{F} \frac{\partial^{2}\left[P_{1}^{F}\right]}{\partial x^{2}}-k_{a}\left[P_{1}^{F}\right]+k_{d}\left[P_{1}^{B}\right], \\
\frac{\partial\left[P_{1}^{B}\right]}{\partial t}=D_{B} \frac{\partial^{2}\left[P_{1}^{B}\right]}{\partial x^{2}}+k_{a}\left[P_{1}^{F}\right]-k_{d}\left[P_{1}^{B}\right],
\end{gathered}
$$

where brackets denote concentration.

Equation (10) can be solved in Fourier space to predict characteristic frequencies of the system as a function of the wave vector $q$, equivalent to the relaxation time $\langle\tau\rangle$ for each $d$ spacing measured by FRS [36]. Fitting the tracer diffusion data to the two-state model enables quantification of the characteristic association and dissociation rate constants ( $k_{a}$ and $k_{d}$, respectively) of the $P_{1}$ tracer, which are expected to reflect the kinetics of all such coiled-coil domains in the network. As shown in Fig. 7, the $P_{1}$ diffusion data are well captured by the two-state model for both gel concentrations, including both the apparent superdiffusive and terminal Fickian regimes. The fits reveal $P_{1}$ dissociation times (given by $\tau_{d}=k_{d}^{-1}$ ) of $\tau_{d, 6.5 \%}=17 \pm 3 \mathrm{~s}$ in the $6.5 \%$ gel and $\tau_{d, 15 \%}=$ $1000 \pm 200 \mathrm{~s}$ in the $15 \%$ gel, where uncertainties are $95 \%$ confidence intervals. While the $6.5 \%$ gel's dissociation time is similar to both the limiting time scale governing exit from the caging regime from self-diffusion measurements $\left[\tau^{*} \approx\right.$
6-12 s; Fig. 6(a)] and the coiled-coil exchange time measured by rheology $\left(\tau_{\text {rheo }}=2.4 \mathrm{~s}\right.$; Fig. S5 [44] and Ref. [36]), the large dissociation time of the $15 \%$ gel reveals an unexpectedly strong concentration dependence of the coiled-coil dissociation kinetics. In addition, the diffusion length scales corresponding to $P_{1}$ dissociation (i.e., the inflection point of the curves) are $d_{\text {inflec, } 6.5 \%}^{2}=1.4 \mu \mathrm{m}^{2}$ and $d_{\text {inflec, } 15 \%}^{2}=2.7 \mu \mathrm{m}^{2}$ for the $6.5 \%$ and $15 \%$ gels, respectively. These length scales are roughly two orders of magnitude greater than the $P_{1}$ radius of gyration $(\sim 7.8 \mathrm{~nm})$, revealing surprisingly high mobility of $P_{1}$ tracers in their bound state before unbinding.

The unexpected concentration dependence of $\tau_{d}$ and similarity between $P_{1}$ and $P_{4}$ diffusion times may be explained by the presence of multichain clusters within the gel that exhibit cooperative diffusion on longer length scales. $P_{1}$ tracers may be trapped within larger $P_{4}$ clusters that constrain their motion to resemble $P_{4}$ self-diffusion, especially at higher gel concentration. These clusters may behave similarly to other self-interacting systems with long-range correlations such as living polymers and wormlike micelles [76], imparting $P_{1}$ chains with walkinglike modes and other forms of bound mobility not captured in the single-chain formulation of the two-state model. The calculated $P_{1}$ dissociation times would thus reflect the time for complete disengagement of these clusters from the network, which would require dissociation of multiple $P$ domains. The presence of loops in the network may also enhance the similarity between $P_{1}$ and $P_{4}$ dynamics by decreasing the number of sticker dissociation events required for $P_{4}$ chains (or clusters) to hop. However, further study is required to explain the mechanisms behind the similarity between $P_{1}$ and $P_{4}$ diffusion times observed here, as well as the relationship between the $P_{1}$ dissociation times obtained from tracer diffusion (Fig. 7) and the hypothesized intrinsic coiledcoil dissociation time inferred from $P_{4}$ self-diffusion (Fig. 6).

\section{CONCLUSION}

Using neutron spin-echo spectroscopy and forced Rayleigh scattering, chain dynamics in highly associating systems are found to be governed by a complex interplay between segmental relaxation and transient binding, resulting in several dynamic regimes spanning molecular to mesoscopic length scales. The transition from segmental relaxation to centerof-mass diffusion is bridged by a caging regime on a length scale comparable to the junction spacing and a time scale governed by a hypothesized concentration-invariant sticker dissociation time. Multiple regimes of apparent superdiffusion are observed on mesoscopic length scales, attributed to transitions between various diffusive modes, including hopping of chains or clusters. Finally, single-sticker diffusion measurements suggest the importance of correlated cluster motion on large length scales, which has often been neglected in previous analyses [28,33,34,79]. The findings of this work provide insight into the relationship between submolecular relaxation and center-of-mass diffusion in associating systems across a range of length scales and may be generalized to other crowded systems with supramolecular interactions, enabling the design of novel soft materials with tailored transport properties. 


\section{ACKNOWLEDGMENTS}

Forced Rayleigh scattering experiments were supported by the National Science Foundation (Award No. DMR1709315), and neutron scattering experiments were supported by the Department of Energy (Award No. DE-SC0007106). A.R. acknowledges support from the Department of De- fense through a National Defense Science and Engineering Graduate Fellowship. The authors thank R. Bataglioli for assistance with molecular cloning; W.-R. Chen, C. Do, and C. Gao for assistance with SANS measurements, and P. Zolnierczuk, M. Odom, and $\mathrm{H}$. Beech for assistance with NSE measurements.

A.R. and H.Y. contributed equally to this work.
[1] F. Hofling and T. Franosch, Anomalous transport in the crowded world of biological cells, Rep. Prog. Phys. 76, 046602 (2013).

[2] L. Masaro and X. X. Zhu, Physical models of diffusion for polymer solutions, gels and solids, Prog. Polym. Sci. 24, 731 (1999).

[3] W. A. Petka, J. L. Harden, K. P. McGrath, D. Wirtz, and D. A. Tirrell, Reversible hydrogels from self-assembling artificial proteins, Science 281, 389 (1998).

[4] T. Aida, E. W. Meijer, and S. I. Stupp, Functional Supramolecular Polymers, Science 335, 813 (2012).

[5] A. Harada, R. Kobayashi, Y. Takashima, A. Hashidzume, and H. Yamaguchi, Macroscopic self-assembly through molecular recognition, Nat. Chem. 3, 34 (2011).

[6] D. S. Banks and C. Fradin, Anomalous diffusion of proteins due to molecular crowding, Biophys. J. 89, 2960 (2005).

[7] M. J. Glassman, J. Chan, and B. D. Olsen, Reinforcement of shear thinning protein hydrogels by responsive block copolymer self-assembly, Adv. Funct. Mater. 23, 1182 (2013).

[8] M. Carpineti and M. Giglio, Transition from Semiorder to Disorder in the Aggregation of Dense Colloidal Solutions, Phys. Rev. Lett. 70, 3828 (1993).

[9] M. Weiss, M. Elsner, F. Kartberg, and T. Nilsson, Anomalous subdiffusion is a measure for cytoplasmic crowding in living cells, Biophys. J. 87, 3518 (2004).

[10] Y. Gu, J. Zhao, and J. A. Johnson, A (Macro)molecular-level understanding of polymer network topology, Trends Chem. 1, 318 (2019).

[11] E. N. de Azevedo, P. L. de Sousa, R. E. de Souza, M. Engelsberg, N. M. Mde, and M. A. Silva, Concentrationdependent diffusivity and anomalous diffusion: A magnetic resonance imaging study of water ingress in porous zeolite, Phys. Rev. E 73, 011204 (2006).

[12] E. Zaccarelli and W. C. Poon, Colloidal glasses and gels: the interplay of bonding and caging, Proc. Natl. Acad. Sci. USA 106, 15203 (2009).

[13] S. P. Zustiak, R. Nossal, and D. L. Sackett, Hindered diffusion in polymeric solutions studied by fluorescence correlation spectroscopy, Biophys. J. 101, 255 (2011).

[14] M. J. Saxton, Anomalous diffusion due to obstacles: a monte carlo study, Biophys. J. 66, 394 (1994).

[15] H. Sanabria, Y. Kubota, and M. N. Waxham, Multiple diffusion mechanisms due to nanostructuring in crowded environments, Biophys. J. 92, 313 (2007).

[16] S. Frey, R. P. Richter, and D. Gorlich, FG-Rich repeats of nuclear pore proteins form a three-dimensional meshwork with hydrogel-like properties, Science 314, 815 (2006).

[17] M. Kim, W. G. Chen, J. W. Kang, M. J. Glassman, K. Ribbeck, and B. D. Olsen, Artificially engineered protein hydrogels adapted from the nucleoporin Nsp1 for selective biomolecular transport, Adv. Mater. 27, 4207 (2015).

[18] W. Shen, K. Zhang, J. A. Kornfield, and D. A. Tirrell, Tuning the erosion rate of artificial protein hydrogels through control of network topology, Nat. Mater. 5, 153 (2006).

[19] K. Ribbeck and D. Gorlich, Kinetic analysis of translocation through nuclearpore complexes, EMBO J. 20, 1320 (2001).

[20] Y. J. Yang, D. J. Mai, T. J. Dursch, and B. D. Olsen, Nucleopore-inspired polymer hydrogels for selective biomolecular transport, Biomacromolecules 19, 3905 (2018).

[21] M. Kim, W. G. Chen, B. S. Souza, and B. D. Olsen, Selective biomolecular separation system inspired by the nuclear pore complex and nuclear transport, Mol. Syst. Des. Eng. 2, 149 (2017).

[22] B. D. Olsen, J. A. Kornfield, and D. A. Tirrell, Yielding behavior in injectable hydrogels from telechelic proteins, Macromolecules 43, 9094 (2010).

[23] M. Guo, L. M. Pitet, H. M. Wyss, M. Vos, P. Y. Dankers, and E. W. Meijer, Tough stimuli-responsive supramolecular hydrogels with hydrogen-bonding network junctions, J. Am. Chem. Soc. 136, 6969 (2014).

[24] N. Holten-Andersen, M. J. Harrington, H. Birkedal, B. P. Lee, P. B. Messersmith, K. Y. Lee, and J. H. Waite, pH-induced metalligand cross-links inspired by mussel yield self-healing polymer networks with near-covalent elastic moduli, Proc. Natl. Acad. Sci. USA 108, 2651 (2011).

[25] C. H. Li et al., A highly stretchable autonomous self-healing elastomer, Nat. Chem. 8, 618 (2016).

[26] P. Cordier, F. Tournilhac, C. Soulie-Ziakovic, and L. Leibler, Self-healing and thermoreversible rubber from supramolecular assembly, Nature (London) 451, 977 (2008).

[27] R. Wang, M. K. Sing, R. K. Avery, B. S. Souza, M. Kim, and B. D. Olsen, Classical challenges in the physical chemistry of polymer networks and the design of new materials, Acc. Chem. Res. 49, 2786 (2016).

[28] P. B. Rapp, A. K. Omar, B. R. Silverman, Z. G. Wang, and D. A. Tirrell, Mechanisms of diffusion in associative polymer networks: evidence for chain hopping, J. Am. Chem. Soc. 140, 14185 (2018).

[29] E. B. Stukalin, L. H. Cai, N. A. Kumar, L. Leibler, and M. Rubinstein, Self-Healing of unentangled polymer networks with reversible bonds, Macromolecules 46, 7525 (2013).

[30] S. Seiffert and J. Sprakel, Physical chemistry of supramolecular polymer networks, Chem. Soc. Rev. 41, 909 (2012).

[31] M. Rubinstein and A. N. Semenov, Thermoreversible gelation in solutions of associating polymers. 2. linear dynamics, Macromolecules 31, 1386 (1997). 
[32] W. C. Yount, D. M. Loveless, and S. L. Craig, Small-molecule dynamics and mechanisms underlying the macroscopic mechanical properties of coordinatively cross-linked polymer networks, J. Am. Chem. Soc. 127, 14488 (2005).

[33] P. B. Rapp, A. K. Omar, J. J. Shen, M. E. Buck, Z. G. Wang, and D. A. Tirrell, Analysis and control of chain mobility in protein hydrogels, J. Am. Chem. Soc. 139, 3796 (2017).

[34] J. Ramirez, T. J. Dursch, and B. D. Olsen, A molecular explanation for anomalous diffusion in supramolecular polymer networks, Macromolecules 51, 2517 (2018).

[35] W. V. Broek and N. L. Thompson, When bivalent proteins might walk across cell surfaces, J. Phys. Chem. 100, 11471 (1996).

[36] S. Tang, M. Wang, and B. D. Olsen, Anomalous self-diffusion and sticky Rouse dynamics in associative protein hydrogels, J. Am. Chem. Soc. 137, 3946 (2015).

[37] S. Tang, A. Habicht, S. Li, S. Seiffert, and B. D. Olsen, Selfdiffusion of associating star-shaped polymers, Macromolecules 49, 5599 (2016).

[38] J. T. Holthausen, C. Wyman, and R. Kanaar, Regulation of DNA strand exchange in homologous recombination, DNA Repair (Amst.) 9, 1264 (2010).

[39] Z. Bu, R. Biehl, M. Monkenbusch, D. Richter, and D. J. Callaway, Coupled protein domain motion in Taq polymerase revealed by neutron spin-echo spectroscopy, Proc. Natl. Acad. Sci. USA 102, 17646 (2005).

[40] J. O. Wrabl, J. Gu, T. Liu, T. P. Schrank, S. T. Whitten, and V. J. Hilser, The role of protein conformational fluctuations in allostery, function, and evolution, Biophys. Chem. 159, 129 (2011).

[41] M. A. Stuart et al., Emerging applications of stimuli-responsive polymer materials, Nat. Mater. 9, 101 (2010).

[42] M. J. Webber, E. A. Appel, E. W. Meijer, and R. Langer, Supramolecular biomaterials, Nat. Mater. 15, 13 (2016).

[43] X. Yan, F. Wang, B. Zheng, and F. Huang, Stimuli-responsive supramolecular polymeric materials, Chem. Soc. Rev. 41, 6042 (2012).

[44] See Supplemental Material at http://link.aps.org/supplemental/ 10.1103/PhysRevResearch.2.043369 for additional details of experimental procedures and data analysis related to materials synthesis, shear rheology, small-angle neutron scattering, neutron spin-echo spectroscopy, and forced Rayleigh scattering.

[45] J. Fiaux, E. B. Bertelsen, A. L. Horwich, and K. Wuthrich, Uniform and residue-specific $15 \mathrm{~N}$-labeling of proteins on a highly deuterated background, J. Biomol. NMR 29, 289 (2004).

[46] M. Wang, K. Timachova, and B. D. Olsen, Experimental measurement of coil-rod-coil block copolymer tracer diffusion through entangled coil homopolymers, Macromolecules 46, 1651 (2013).

[47] M. Wang, K. Timachova, and B. D. Olsen, Self-diffusion and constraint release in isotropic entangled rod-coil block copolymers, Macromolecules 48, 3121 (2015).

[48] O. Arnold et al., Mantid-Data analysis and visualization package for neutron scattering and SR experiments, Nucl. Instrum. Methods Phys. Res. A 764, 156 (2014).

[49] Mantid (2013): Manipulation and Analysis Toolkit for Instrument Data, 2013.

[50] R. Navarro Pérez, J. E. Amaro, and E. Ruiz Arriola, Bootstrapping the statistical uncertainties of NN scattering data, Phys. Lett. B 738, 155 (2014).
[51] L. F. Shampine, Vectorized adaptive quadrature in MATLAB, J. Comput. Appl. Math. 211, 131 (2008).

[52] V. N. Malashkevich, R. A. Kammerer, V. P. Efimov, T. Schulthess, and J. Engel, The crystal structure of a five-stranded coiled coil in comp: A prototype ion channel? Science 274, 761 (1996).

[53] S. B. Kennedy, K. Littrell, P. Thiyagarajan, D. A. Tirrell, and T. P. Russell, Controlled structure in artificial protein hydrogels, Macromolecules 38, 7470 (2005).

[54] B. Hammouda, Probing Nanoscale Structures: The SANS Toolbox (NIST, Gaithersburg, MD, 2010).

[55] C. E. R. Edwards, D. J. Mai, S. Tang, and B. D. Olsen, Molecular anisotropy and rearrangement as mechanisms of toughness and extensibility in entangled physical gels, Phys. Rev. Materials 4, 015602 (2020).

[56] R. A. Hule, R. P. Nagarkar, B. Hammouda, J. P. Schneider, and D. J. Pochan, Dependence of self-assembled peptide hydrogel network structure on local fibril nanostructure, Macromolecules 42, 7137 (2009).

[57] E. M. Saffer, M. A. Lackey, D. M. Griffin, S. Kishore, G N. Tew, and S. R. Bhatia, SANS study of highly resilient poly(ethylene glycol) hydrogels, Soft Matter 10, 1905 (2014).

[58] T. Matsunaga, T. Sakai, Y. Akagi, U.-I. Chung, and M. Shibayama, SANS and SLS studies on tetra-arm PEG Gels in as-prepared and swollen states, Macromolecules 42, 6245 (2009).

[59] G. Evmenenko, E. Theunissen, K. Mortensen, and H. Reynaers, SANS study of surfactant ordering in $\kappa$-carrageenan/cetylpyridinium chloride complexes, Polymer 42, 2907 (2001).

[60] T. Kanaya, M. Ohkura, and K. Kaji, Structure of poly(vinyl alcohol) gels studied by wide- and small-angle neutron scattering, Macromolecules 27, 5609 (1994).

[61] S. Mallam, F. Horkay, A. M. Hecht, A. A. Rennie, and E. Geissler, Microscopic and macroscopic thermodynamic observations in swollen poly(dimethylsiloxane) networks, Macromolecules 24, 543 (1991).

[62] M. Shibayama, T. Tanaka, and C. C. Han, Small angle neutron scattering study on poly(N-isopropyl acrylamide) gels near their volume-phase transition temperature, J. Chem. Phys. 97, 6829 (1992).

[63] B. Hammouda, D. L. Ho, and S. Kline, Insight into clustering in poly(ethylene oxide) solutions, Macromolecules 37, 6932 (2004).

[64] F. Horkay and B. Hammouda, Small-angle neutron scattering from typical synthetic and biopolymer solutions, Colloid Polym. Sci. 286, 611 (2008).

[65] W. Shen, J. A. Kornfield, and D. A. Tirrell, Structure and mechanical properties of artificial protein hydrogels assembled through aggregation of leucine zipper peptide domains, Soft Matter 3, 99 (2007).

[66] P.-G. de Gennes, Scaling Concepts in Polymer Physics (Cornell University Press, Ithaca, 1979).

[67] D. Richter, M. Monkenbusch, J. Allgeier, A. Arbe, J. Colmenero, B. Farago, Y. Cheol Bae, and R. Faust, From Rouse dynamics to local relaxation: A neutron spin echo study on polyisobutylene melts, J. Chem. Phys. 111, 6107 (1999).

[68] A. Ghavami, H. Kobayashi, and R. G. Winkler, Internal dynamics of microgels: A mesoscale hydrodynamic simulation study, J. Chem. Phys. 145, 244902 (2016). 
[69] M. Doi and S. F. Edwards, The Theory of Polymer Dynamics (Oxford University Press, New York, 1986).

[70] S. Koizumi, M. Monkenbusch, D. Richter, D. Schwahn, and B. Farago, Concentration fluctuations in polymer gel investigated by neutron scattering: static inhomogeneity in swollen gel, J. Chem. Phys. 121, 12721 (2004).

[71] T. Tanaka, L. O. Hocker, and G. B. Benedek, Spectrum of light scattered from a viscoelastic gel, J. Chem. Phys. 59, 5151 (1973).

[72] T. Kanaya, N. Takahashi, K. Nishida, H. Seto, M. Nagao, and T. Takeda, Neutron spin-echo studies on dynamic and static fluctuations in two types of poly(vinyl alcohol) gels, Phys. Rev. E 71, 011801 (2005).

[73] S. Maccarrone, C. Scherzinger, O. Holderer, P. Lindner, M. Sharp, W. Richtering, and D. Richter, Cononsolvency effects on the structure and dynamics of microgels, Macromolecules 47, 5982 (2014).
[74] W. Urbach, H. Hervet, and F. Rondelez, On the application of forced Rayleigh light scattering to mass diffusion measurements, J. Chem. Phys. 83, 1877 (1985).

[75] S. Tang and B. D. Olsen, Relaxation processes in supramolecular metallogels based on histidine-nickel coordination bonds, Macromolecules 49, 9163 (2016).

[76] A. Ott, J. P. Bouchaud, D. Langevin, and W. Urbach, Anomalous Diffusion in "Living Polymers": A Genuine Levy Flight? Phys. Rev. Lett. 65, 2201 (1990).

[77] J. P. Bouchaud and A. Georges, Anomalous diffusion in disordered media: Statistical mechanisms, models and physical applications, Phys. Rep. 195, 127 (1990).

[78] R. S. Hoy and G. H. Fredrickson, Thermoreversible associating polymer networks. I. Interplay of thermodynamics, chemical kinetics, and polymer physics, J. Chem. Phys. 131, 224902 (2009).

[79] B. L. Sprague, R. L. Pego, D. A. Stavreva, and J. G. McNally, Analysis of binding reactions by fluorescence recovery after photobleaching, Biophys. J. 86, 3473 (2004). 